Supplement of Earth Syst. Sci. Data, 12, 2223-2259, 2020

प Earth System

https://doi.org/10.5194/essd-12-2223-2020-supplement

(C) Author(s) 2020. This work is distributed under

the Creative Commons Attribution 4.0 License.

(c) (1)

Supplement of

\title{
Updated tropospheric chemistry reanalysis and emission estimates, TCR-2, for 2005-2018
}

\section{Kazuyuki Miyazaki et al.}

Correspondence to: Kazuyuki Miyazaki(kazuyuki.miyazaki@jpl.nasa.gov)

The copyright of individual parts of the supplement might differ from the CC BY 4.0 License. 


\section{Fig. S1: Latitude-pressure cross section of mean ozone concentration (in ppb) obtained from HIPPO aircraft measurements}

(first row), control run (second row), and reanalysis (third row). The relative difference (in \%) between the control run and the observation (fourth row) and between the reanalysis and the observation (fifth row) is also shown. Results are shown for all HIPPO campaigns (from left to right: HIPPO I, 8-30 January 2009; HIPPO II, 31 October to 22 November 2009; HIPPO III, 24 March to 16 April 2010; HIPPO IV, 14 June to 11 July 2011; and HIPPO V, 9 August to 9 September 2011).
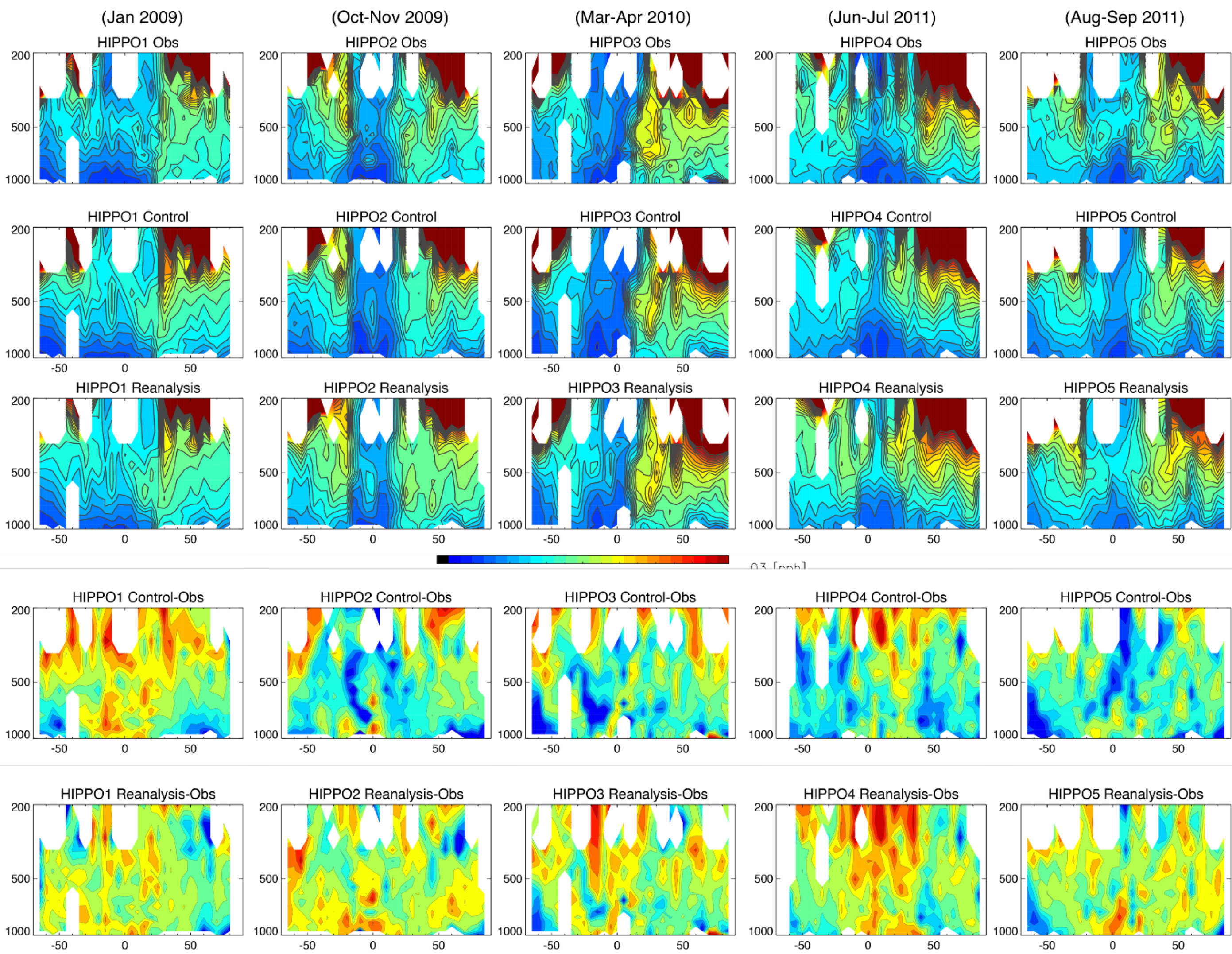
Fig. S2: Same as in Fig. S1, but for CO concentration (in ppb, from first to third row) and its absolute difference (in ppb, from fourth to fifth row) obtained from HIPPO aircraft measurements.
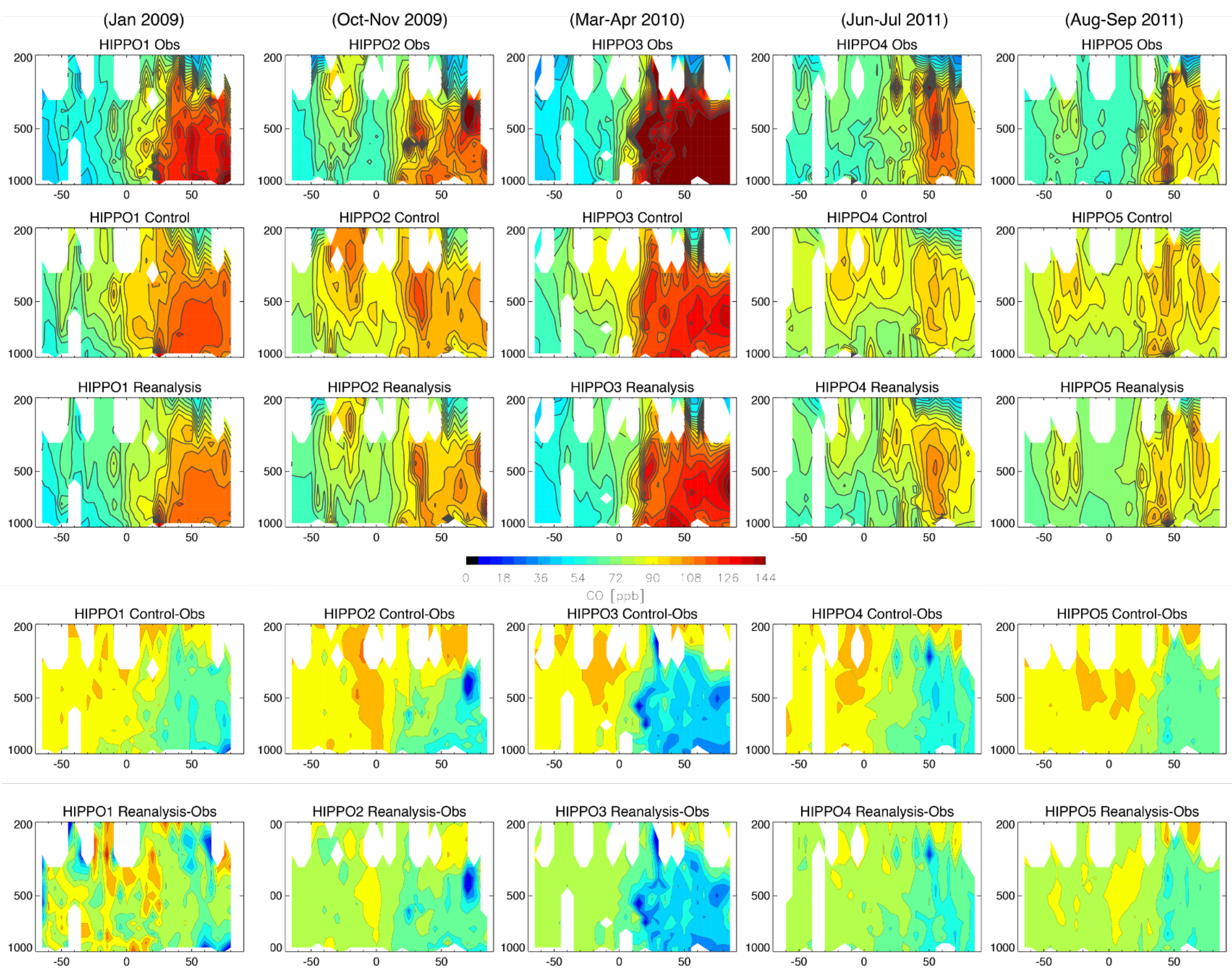
Fig. S3: Anomaly of annual mean tropospheric OH concentration from the 14-year mean (2005-2018) in TCR-2.
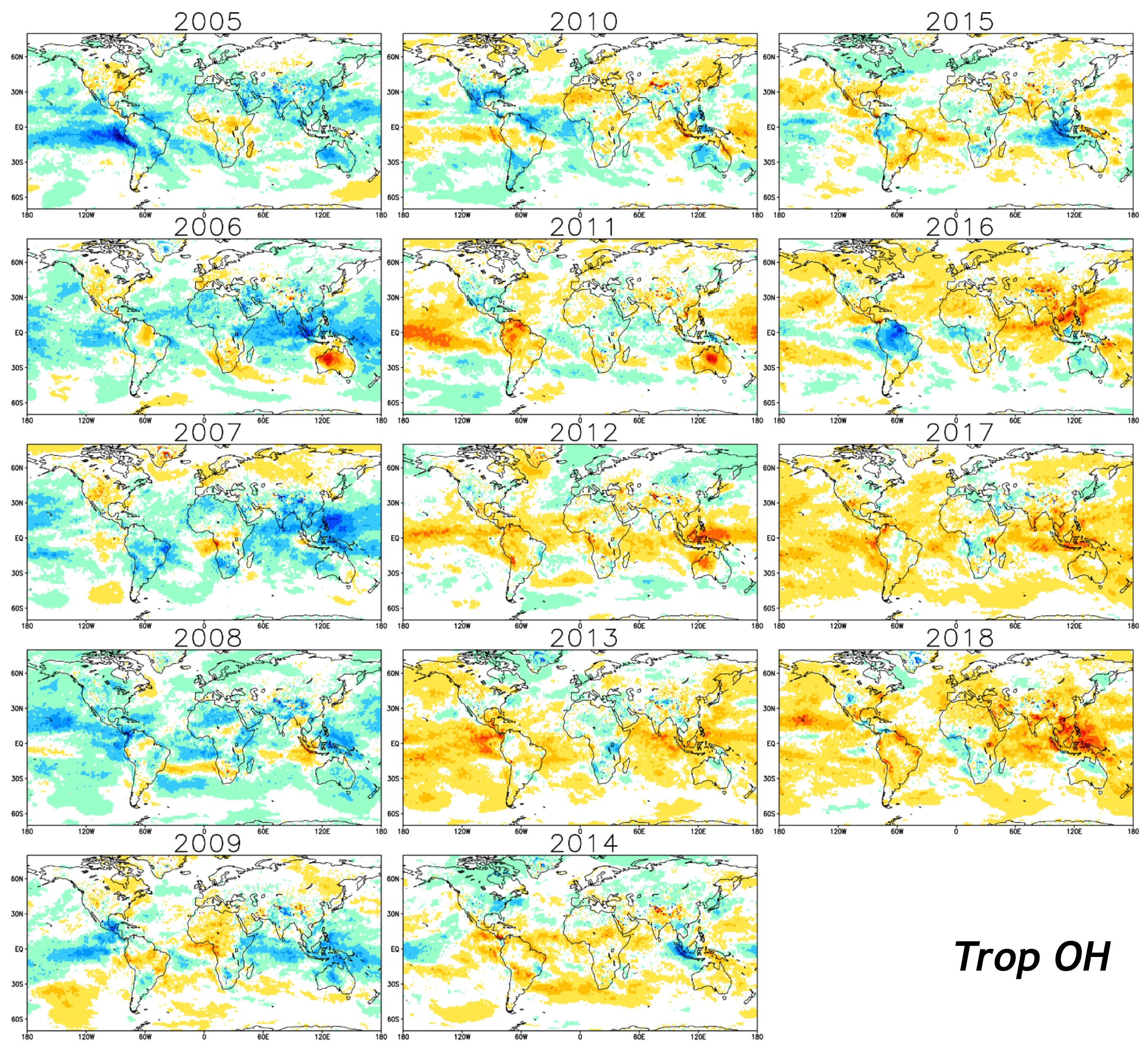

Trop $\mathrm{OH}$ 
Fig. S4: Same as in Fig S3, but for the noTES reanalysis.
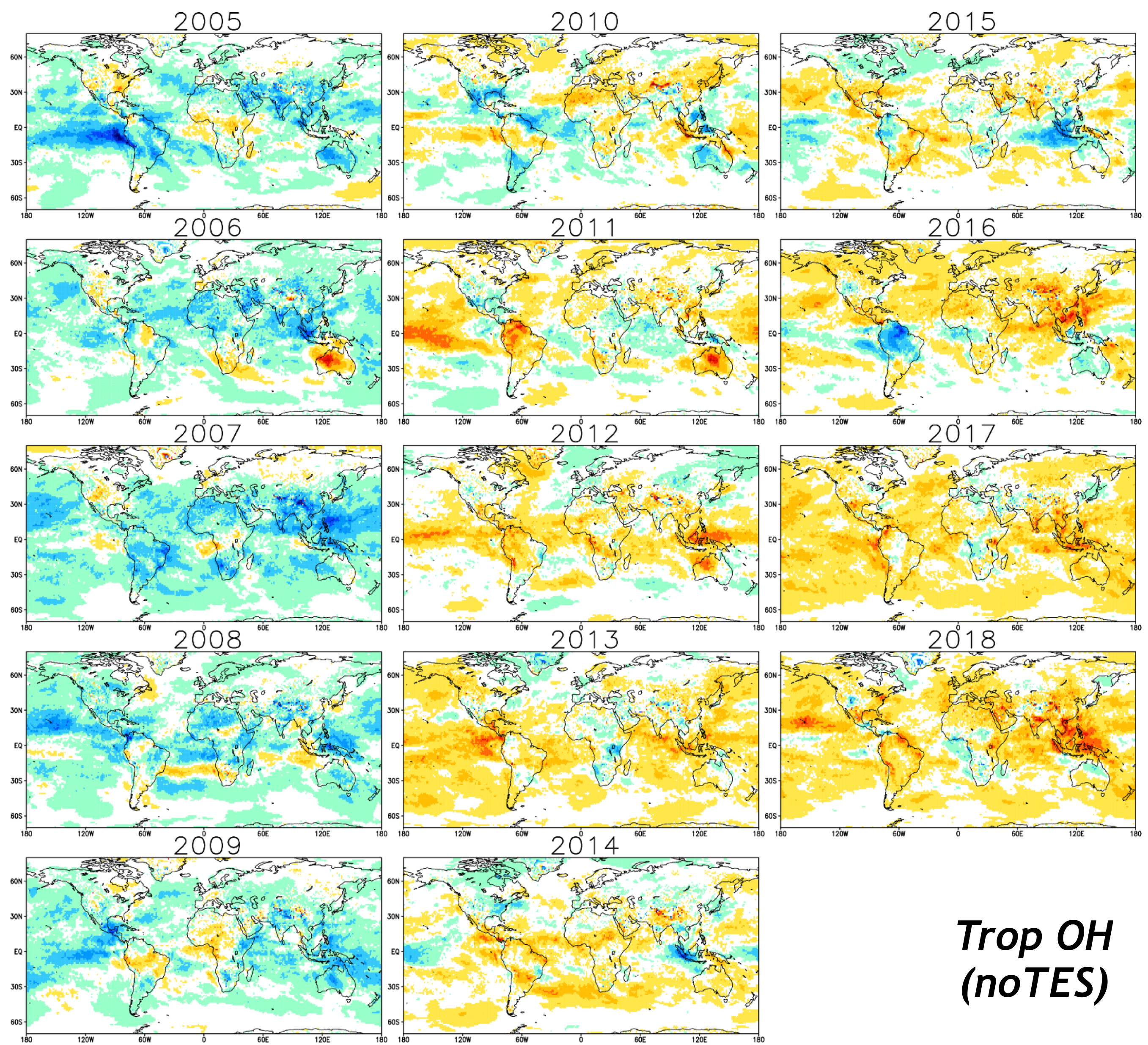

Trop OH (noTES) 
Fig. S5: Vertical profiles of $\mathrm{OH}$ concentrations (pptv) from ATom measurements (black), control run (blue), and chemical reanalysis from ATom-1 in August 2016 and ATom-2 in February 2017.
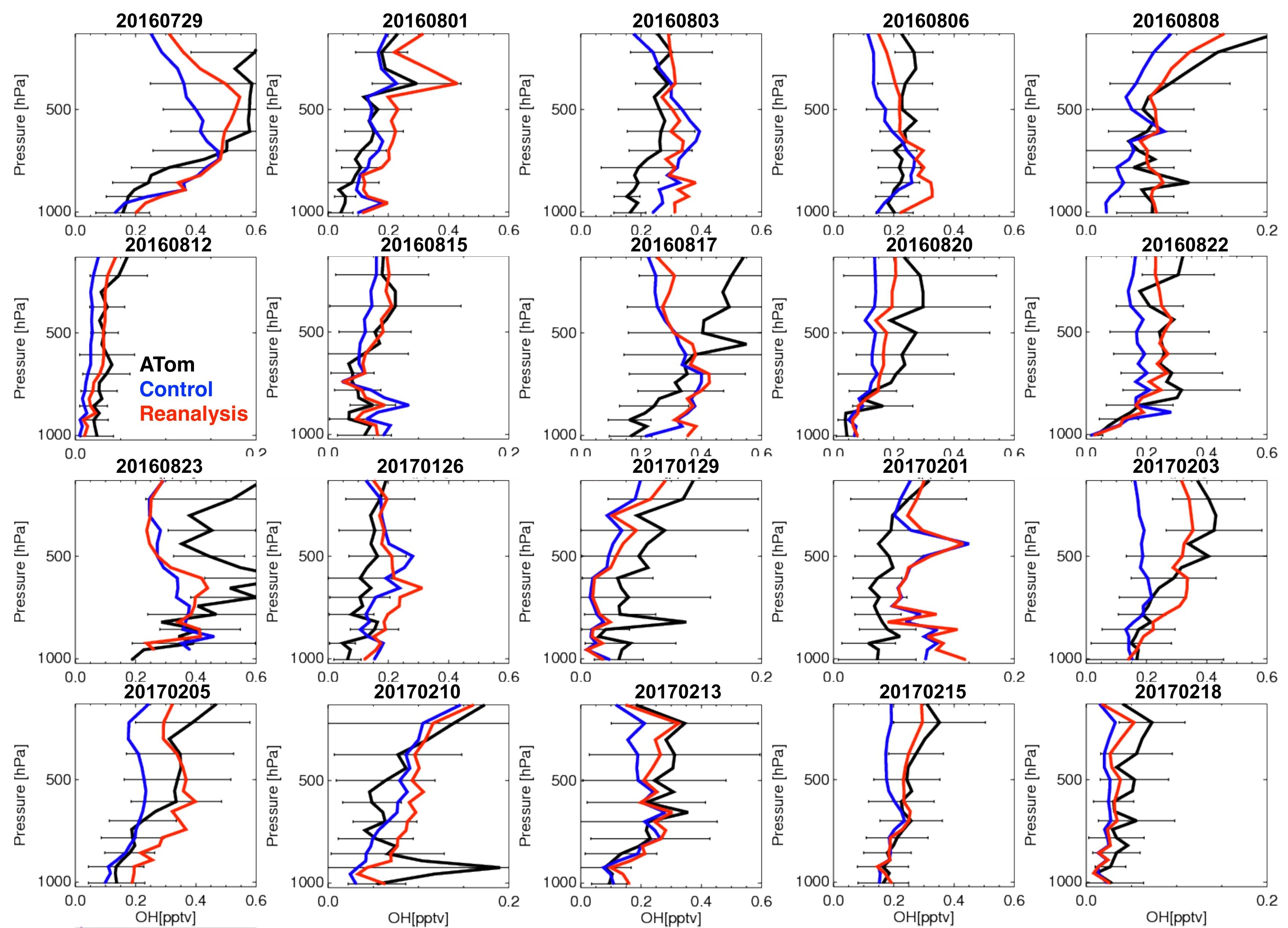
Fig. S6: Anomaly of annual mean surface NOx emissions (in $10^{-11} \mathrm{~kg} \mathrm{~N} \mathrm{~m}^{-2} \mathrm{~s}^{-1}$ ) from the 14 -year mean (2005-2018) in TCR-2.
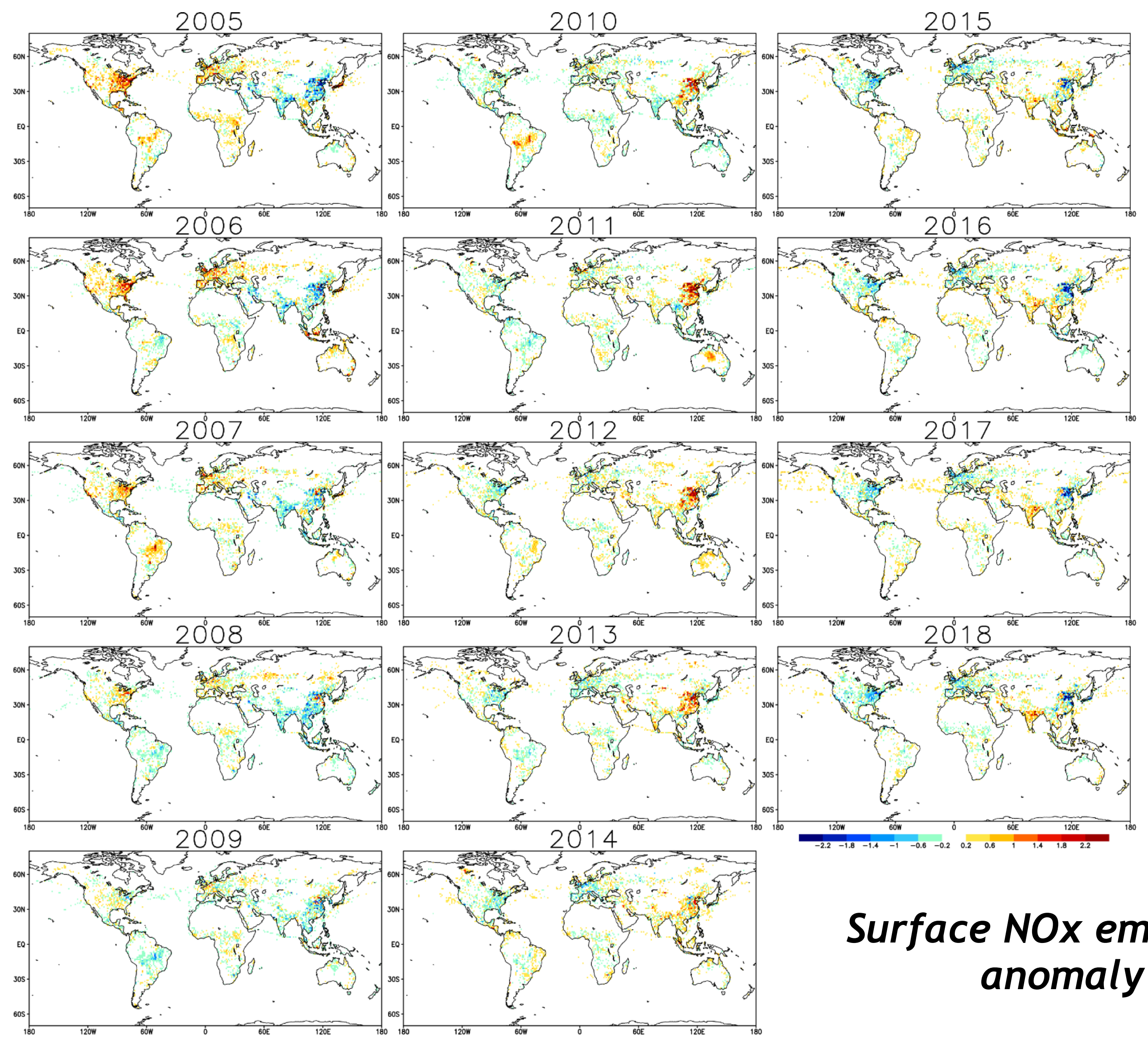

Surface NOx emissions
anomaly

Surface NOx em
anomaly

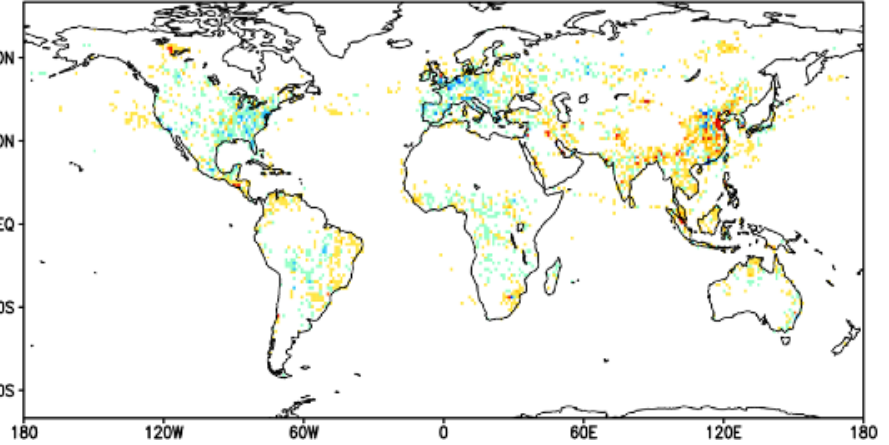


Fig. S7: Anomaly of annual mean surface CO emissions (in $10^{-10} \mathrm{~kg} \mathrm{CO} \mathrm{m}^{-2} \mathrm{~s}^{-1}$ ) from the 14-year mean (2005-2018) in TCR-2.
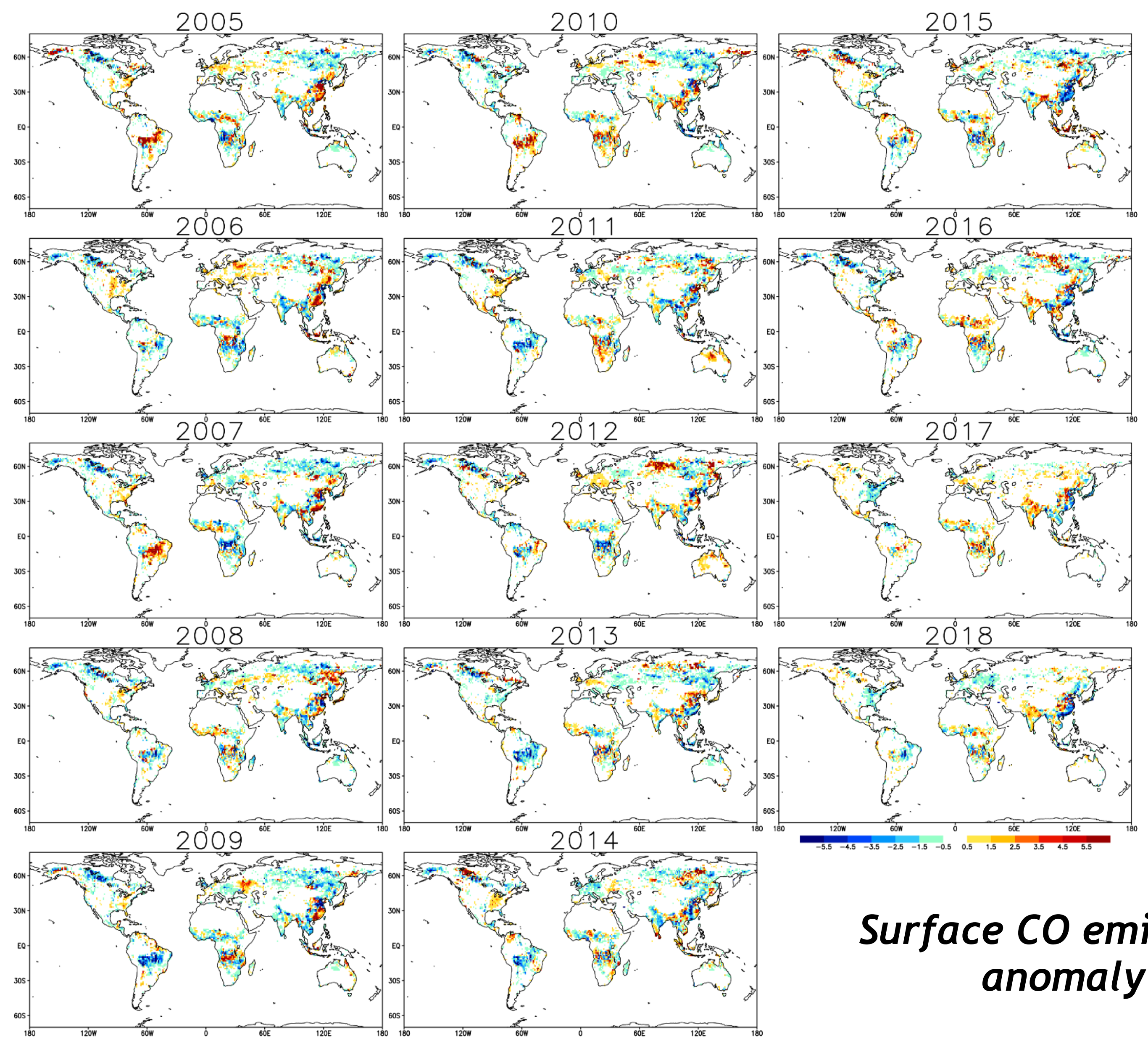

\section{Surface CO emissions anomaly}


Fig. S8: Anomaly of annual mean surface SO2 emissions (in $10^{-12} \mathrm{~kg} \mathrm{~S} \mathrm{~m}^{-2} \mathrm{~s}^{-1}$ ) from the 14 -year mean (2005-2018) in TCR-2.
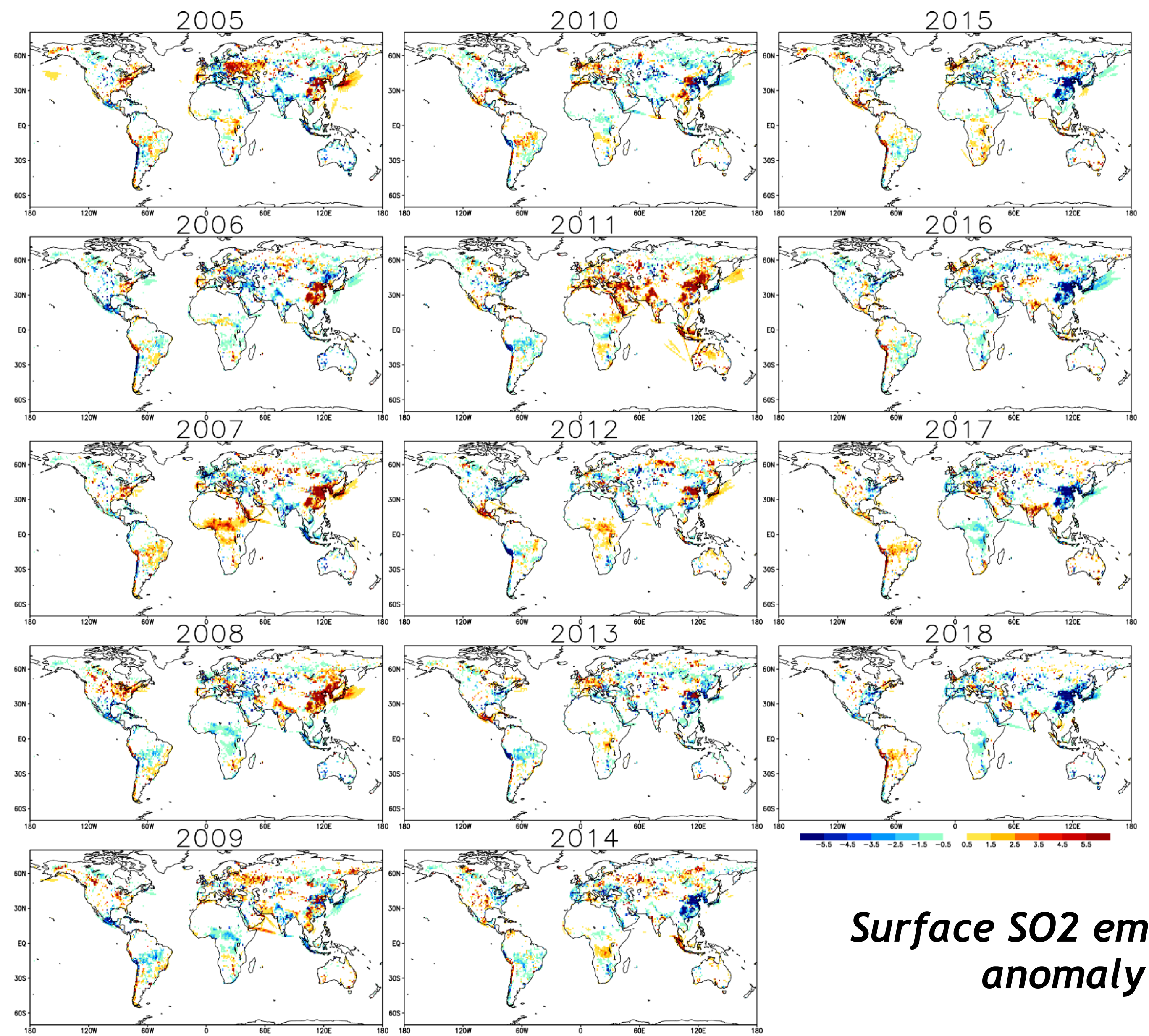

Surface SO2 emissions anomaly 
Fig. S9: Anomaly of annual mean lightning NOx sources (in $10^{-12} \mathrm{~kg} \mathrm{~N} \mathrm{~m}^{-2} \mathrm{~s}^{-1}$ ) from the 14-year mean (2005-2018) in TCR-2.
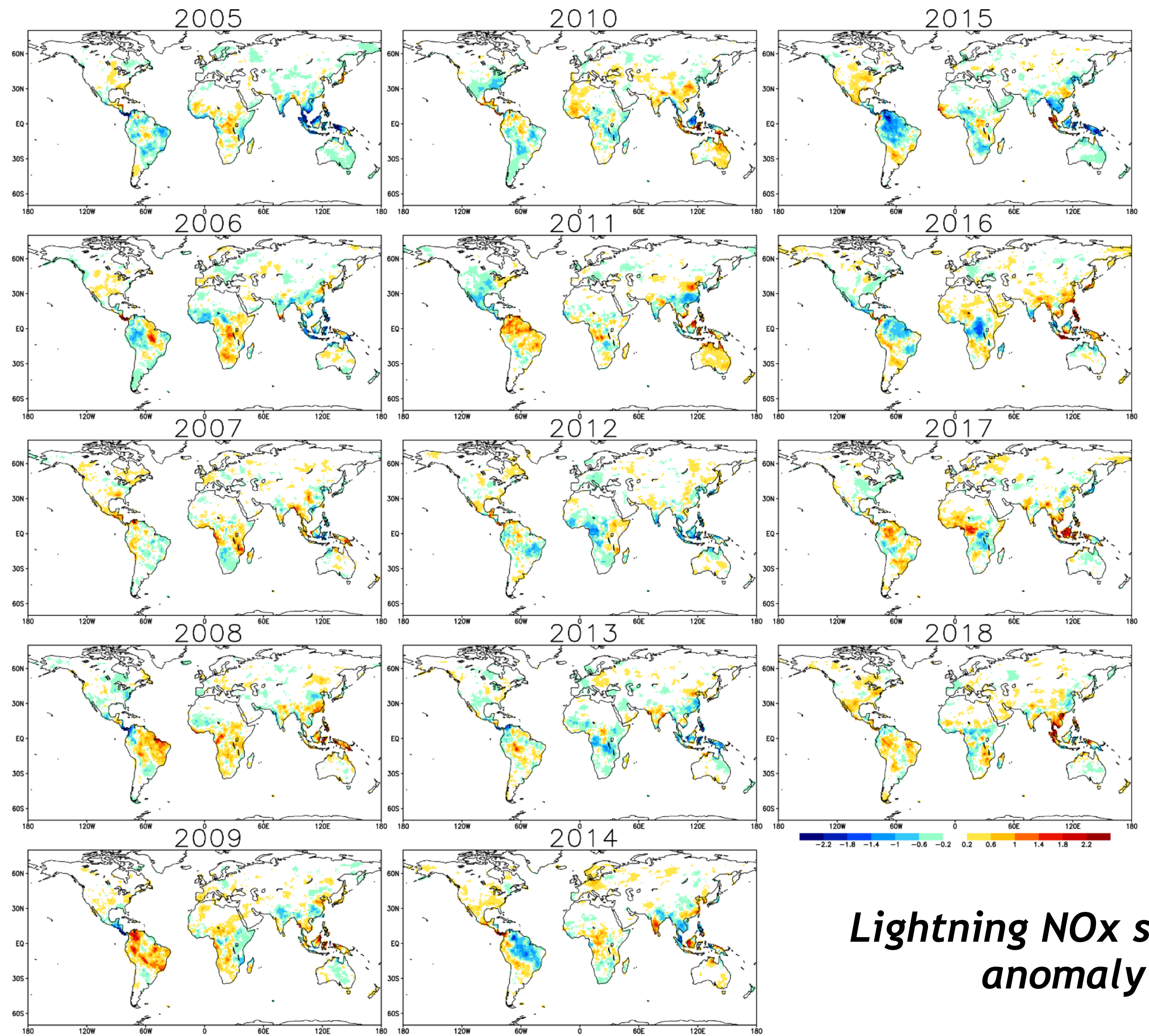

Lightning NOx sources anomaly

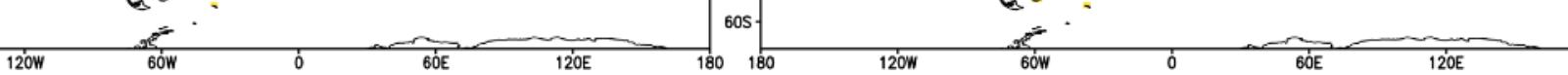


Fig. S10: Anomaly of annual mean surface ozone concentrations (in ppb) from the 14-year mean (2005-2018) in TCR-2.
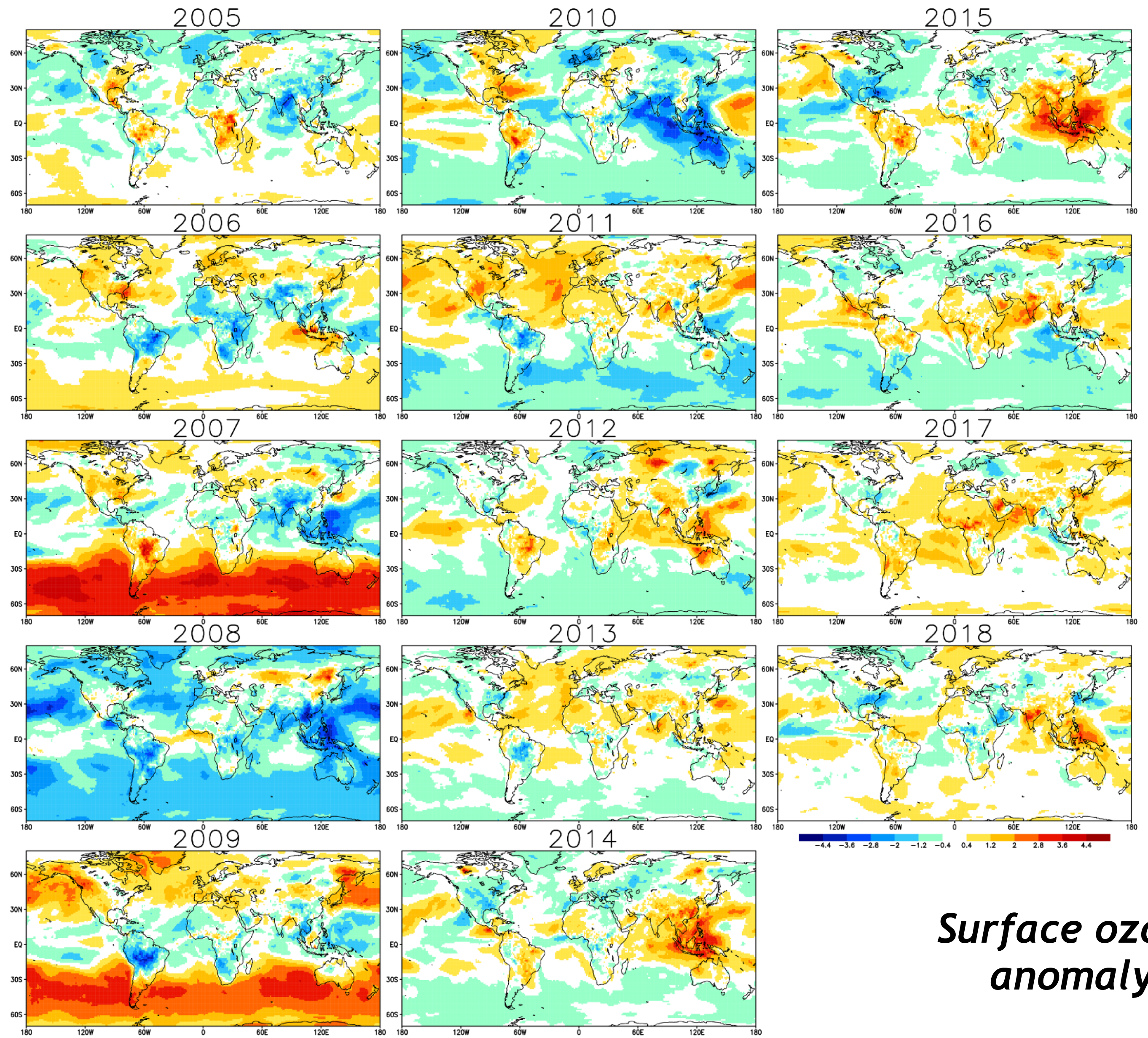

\section{Surface ozone anomaly}


Fig. S11: Same as in Fig S10, but for the noTES reanalysis.
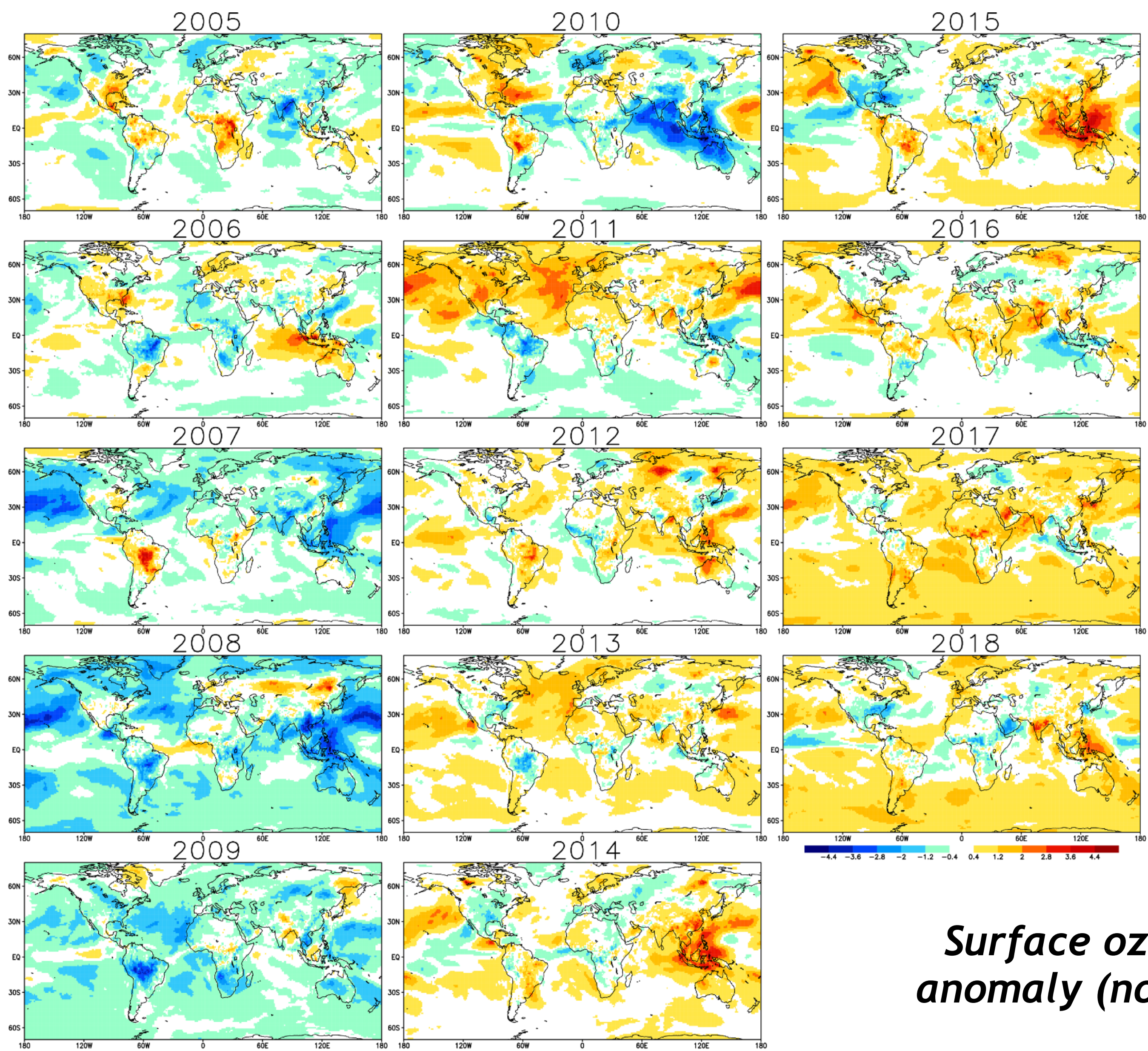

\section{Surface ozone anomaly (noTES)}


Fig. S12: Anomaly of annual mean $500 \mathrm{hPa}$ ozone concentrations (in ppb) from the 14-year mean (2005-2018) in TCR-2.
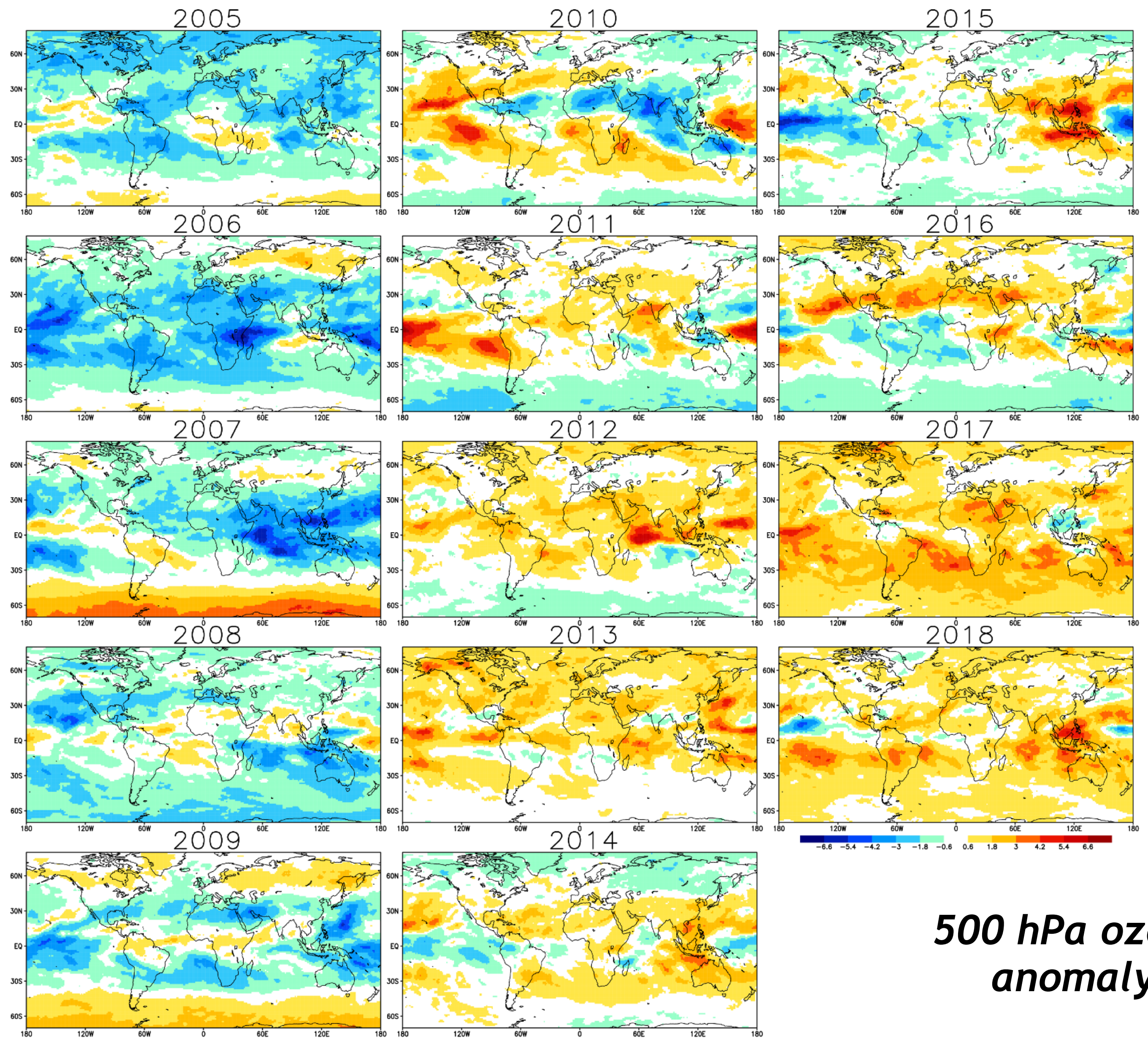

500 hPa ozone anomaly 
Fig. S13: Same as in Fig S12, but for the noTES reanalysis.
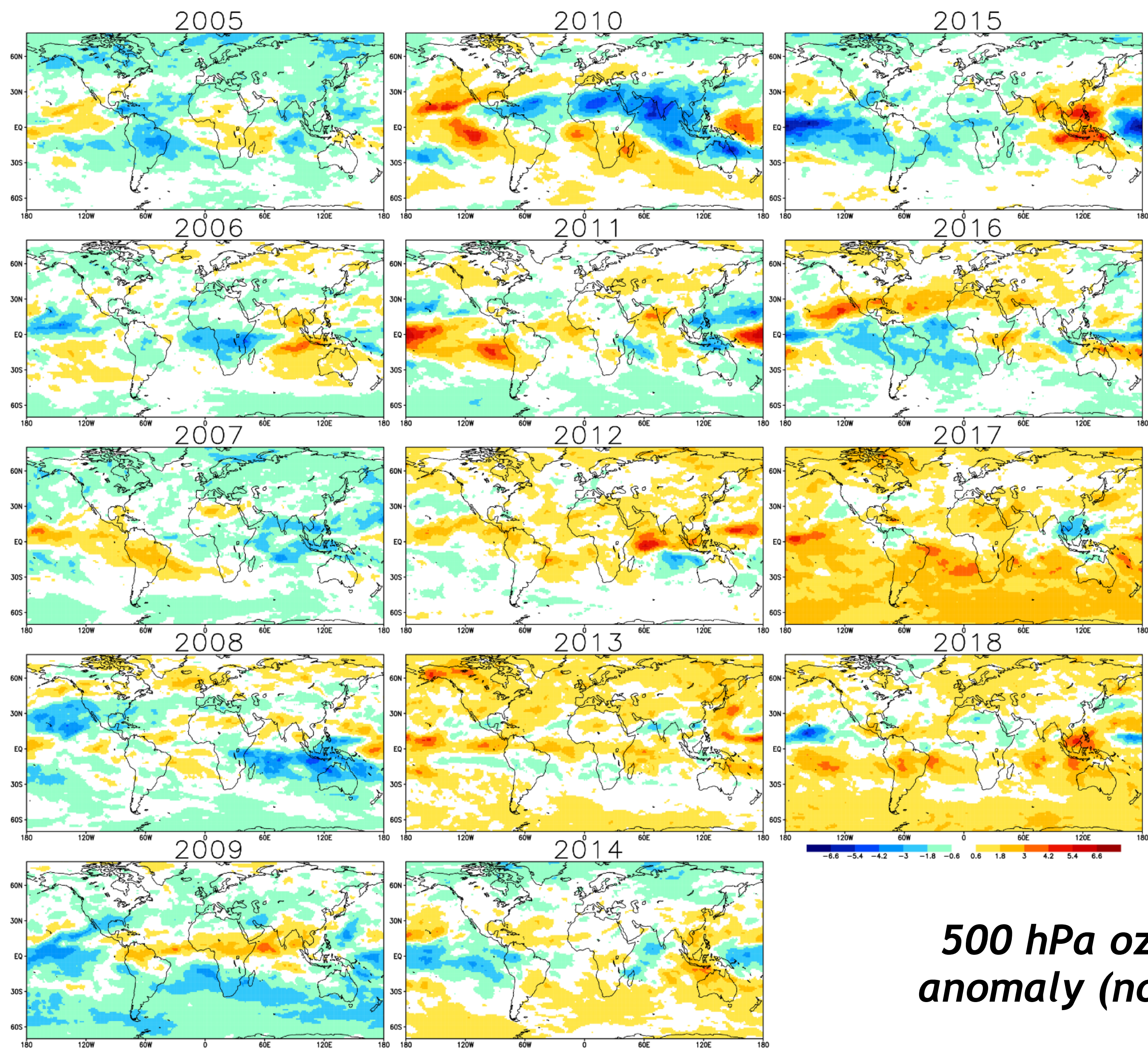

500 hPa ozone anomaly (noTES) 
Fig. S14: Time-latitude cross section of zonal mean analysis spread of ozone (in ppb) at $600 \mathrm{hPa}$ and $200 \mathrm{hPa}$.

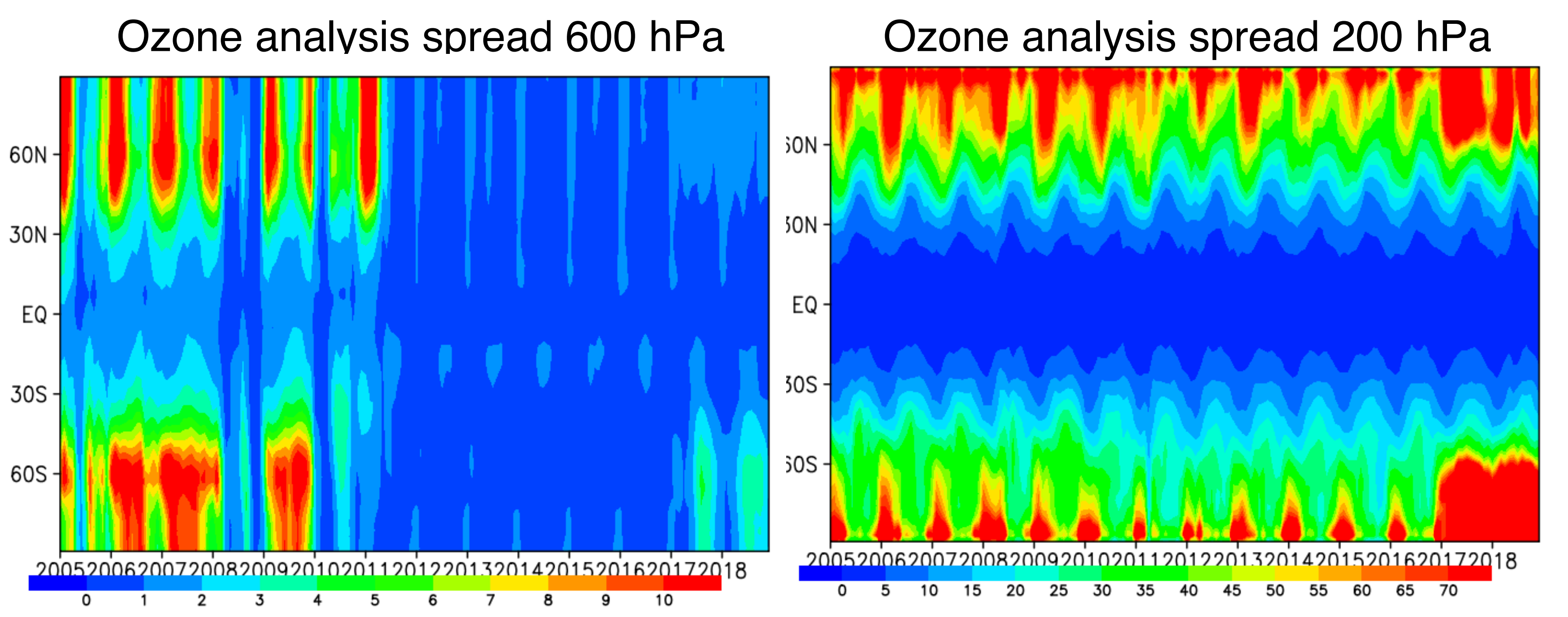


Table S1: Model minus observation comparisons of the mean ozone concentrations(in ppb) between the analysis or control run (in brackets) and the observations for HIPPO aircraft measurements during 2009-2011.

\begin{tabular}{|c|c|c|c|c|c|c|c|c|c|c|c|}
\hline $\begin{array}{l}\text { Ozone } \\
\text { (ppb) }\end{array}$ & & $\begin{array}{l}90-55 S \\
\text { Bias }\end{array}$ & RMSE & $\begin{array}{l}\text { 55-15S } \\
\text { Bias }\end{array}$ & RMSE & $\begin{array}{c}15 \mathrm{~S}-15 \mathrm{~N} \\
\text { Bias }\end{array}$ & RMSE & $\begin{array}{c}15 \mathrm{~N}-55 \mathrm{~N} \\
\text { Bias }\end{array}$ & RMSE & $\begin{array}{c}55 N-90 N \\
\text { Bias }\end{array}$ & RMSE \\
\hline \multirow[t]{2}{*}{$\begin{array}{c}850-500 \\
\mathrm{hPa}\end{array}$} & Assim & -0.9 & 6.1 & 2.0 & 6.6 & 2.4 & 7.4 & 1.6 & 8.7 & 2.0 & 7.7 \\
\hline & Free & -4.5 & 6.2 & -4.1 & 7.2 & -3.6 & 7.5 & -4.6 & 9.5 & -4.0 & 7.3 \\
\hline \multirow[t]{2}{*}{$\begin{array}{c}500-200 \\
\mathrm{hPa}\end{array}$} & Assim & 4.6 & 1.9 & 4.6 & 14.3 & 5.0 & 10.0 & 4.5 & 19.4 & 2.5 & 36.2 \\
\hline & Free & 42.6 & 58.7 & 4.3 & 19.6 & -2.6 & 19.6 & 8.1 & 24.1 & 31.9 & 56.6 \\
\hline
\end{tabular}




\begin{tabular}{|c|c|c|c|c|c|c|c|c|c|c|c|}
\hline $\begin{array}{c}\mathrm{CO} \\
(\mathrm{ppb})\end{array}$ & & $\begin{array}{c}90-55 S \\
\text { Bias }\end{array}$ & RMSE & $\begin{array}{l}\text { 55-15S } \\
\text { Bias }\end{array}$ & RMSE & $\begin{array}{c}15 S-15 N \\
\text { Bias }\end{array}$ & RMSE & $\begin{array}{c}15 \mathrm{~N}-55 \mathrm{~N} \\
\text { Bias }\end{array}$ & RMSE & $\begin{array}{c}55 \mathrm{~N}-90 \mathrm{~N} \\
\text { Bias }\end{array}$ & RMSE \\
\hline \multirow[t]{2}{*}{$\begin{array}{c}850-500 \\
\mathrm{hPa}\end{array}$} & Assim & 6.5 & 6.6 & 6.2 & 7.5 & 3.3 & 9.5 & -9.7 & 16.5 & -15.1 & 17.4 \\
\hline & Free & 17.8 & 17.8 & 18.6 & 18.9 & 15.7 & 18.7 & -5.9 & 16.4 & -15.5 & 16.9 \\
\hline \multirow[t]{2}{*}{$\begin{array}{c}500-200 \\
\mathrm{hPa}\end{array}$} & Assim & 8.7 & 8.9 & 7.9 & 9.4 & 7.1 & 10.1 & -2.4 & 16.5 & -4.0 & 6.5 \\
\hline & Free & 19.9 & 20.0 & 21.6 & 22.1 & 21.7 & 22.4 & 5.0 & 20.0 & -1.2 & 21.4 \\
\hline
\end{tabular}




\section{Table S3: Yearly surface NOx emissions in TgNyr-1.}

\begin{tabular}{|c|c|c|c|c|c|c|c|c|c|c|c|c|c|c|}
\hline TgNyr $^{-1}$ & Globe & $\mathrm{NH}$ & Tropics & $\mathrm{SH}$ & Europe & USA & C Africa & E China & S Africa & $\begin{array}{c}\mathrm{S} \\
\text { America }\end{array}$ & N Africa & SE Asia & Australia & India \\
\hline 2005 & 50.59 & 29.51 & 17.59 & 3.48 & 5.05 & 6.74 & 3.06 & 5.45 & 0.69 & 1.40 & 3.49 & 0.65 & 1.58 & 2.91 \\
\hline 2006 & 50.60 & 30.24 & 16.74 & 3.62 & 5.21 & 6.46 & 2.89 & 5.64 & 0.68 & 1.15 & 3.00 & 0.57 & 1.85 & 3.01 \\
\hline 2007 & 50.39 & 30.04 & 16.65 & 3.70 & 4.98 & 6.07 & 2.80 & 5.83 & 0.71 & 1.46 & 3.26 & 0.62 & 1.57 & 2.92 \\
\hline 2008 & 48.50 & 30.04 & 15.17 & 3.29 & 4.78 & 5.65 & 2.75 & 5.83 & 0.67 & 0.99 & 3.12 & 0.49 & 1.36 & 2.96 \\
\hline 2009 & 47.14 & 28.23 & 15.56 & 3.34 & 4.54 & 5.36 & 2.87 & 5.87 & 0.64 & 0.88 & 3.13 & 0.52 & 1.47 & 3.16 \\
\hline 2010 & 49.83 & 30.23 & 16.29 & 3.31 & 4.57 & 5.08 & 2.87 & 6.77 & 0.67 & 1.56 & 2.91 & 0.61 & 1.25 & 3.11 \\
\hline 2011 & 49.41 & 29.82 & 16.05 & 3.55 & 4.69 & 5.18 & 2.78 & 7.06 & 0.66 & 1.01 & 3.20 & 0.54 & 1.78 & 3.43 \\
\hline 2012 & 49.87 & 28.92 & 17.16 & 3.78 & 4.63 & 5.05 & 2.88 & 6.86 & 0.68 & 1.15 & 3.13 & 0.59 & 1.78 & 3.45 \\
\hline 2013 & 49.87 & 29.82 & 16.67 & 3.39 & 4.42 & 5.05 & 2.83 & 6.84 & 0.67 & 1.00 & 3.10 & 0.59 & 1.52 & 3.50 \\
\hline 2014 & 50.30 & 29.19 & 17.50 & 3.61 & 4.40 & 4.98 & 2.77 & 6.43 & 0.72 & 1.08 & 3.22 & 0.65 & 1.62 & 3.54 \\
\hline 2015 & 49.17 & 27.57 & 17.96 & 3.65 & 4.33 & 4.85 & 2.93 & 5.84 & 0.70 & 1.17 & 3.23 & 0.65 & 1.62 & 3.64 \\
\hline 2016 & 47.93 & 27.39 & 17.19 & 3.35 & 4.47 & 4.84 & 2.84 & 5.63 & 0.68 & 1.05 & 3.30 & 0.67 & 1.45 & 3.66 \\
\hline 2017 & 47.41 & 26.46 & 17.34 & 3.61 & 4.47 & 4.91 & 2.85 & 5.75 & 0.68 & 1.12 & 3.30 & 0.60 & 1.57 & 3.75 \\
\hline 2018 & 48.27 & 27.87 & 16.91 & 3.48 & 4.41 & 4.66 & 2.83 & 5.76 & 0.68 & 1.13 & 3.03 & 0.61 & 1.58 & 3.78 \\
\hline
\end{tabular}




\section{Table S4: Yearly surface CO emissions in TgCOyr-1.}

\begin{tabular}{|c|c|c|c|c|c|c|c|c|c|c|c|c|c|}
\hline TgCOyr $^{-1}$ & Globe & Tropics & $\mathrm{SH}$ & Europe & USA & C Africa & E China & S Africa & $\begin{array}{c}\mathrm{S} \\
\text { America }\end{array}$ & N Africa & SE Asia & Australia & India \\
\hline 2005 & $1159.5: 616.55$ & 503.78 & 39.26 & 47.91 & 67.80 & 156.43 & 195.03 & 7.53 & 79.30 & 107.30 & 14.75 & 11.79 & 73.85 \\
\hline 2006 & $1111.3<612.92$ & 460.56 & 37.88 & 48.94 & 71.27 & 159.31 & 183.60 & 7.10 & 35.76 & 92.31 & 11.28 & 16.99 & 68.19 \\
\hline 2007 & 1108.76589 .86 & 477.70 & 41.22 & 38.30 & 71.19 & 142.79 & 177.46 & 7.68 & 68.18 & 104.14 & 16.68 & 14.27 & 76.75 \\
\hline 2008 & $1058.1: 591.94$ & 429.61 & 36.59 & 40.65 & 69.87 & 164.48 & 174.37 & 8.29 & 30.49 & 112.71 & 10.29 & 9.77 & 74.54 \\
\hline 2009 & $1022.1 ; 549.56$ & 433.24 & 39.38 & 38.55 & 63.43 & 172.43 & 176.35 & 6.70 & 10.06 & 95.46 & 10.91 & 15.52 & 70.61 \\
\hline 2010 & $1152.8\{577.29$ & 530.65 & 44.96 & 41.97 & 56.93 & 186.01 & 179.26 & 8.40 & 86.27 & 102.68 & 17.59 & 8.66 & 79.78 \\
\hline 2011 & $1088.5 \div 583.90$ & 457.03 & 47.68 & 41.65 & 73.54 & 174.98 & 176.92 & 8.64 & 23.87 & 110.75 & 10.53 & 25.58 & 73.81 \\
\hline 2012 & $1130.6: 638.17$ & 448.83 & 43.64 & 49.94 & 67.46 & 150.93 & 179.53 & 7.94 & 32.26 & 106.95 & 13.31 & 26.85 & 80.96 \\
\hline 2013 & $1065.0: 591.91$ & 430.82 & 42.29 & 43.38 & 57.67 & 168.20 & 183.18 & 8.71 & 12.67 & 105.12 & 14.83 & 12.34 & 76.58 \\
\hline 2014 & 1102.37597 .61 & 467.14 & 37.63 & 34.96 & 69.74 & 164.90 & 168.54 & 9.03 & 19.09 & 102.23 & 11.70 & 14.94 & 73.35 \\
\hline 2015 & 1137.977 . 93 . & 524.99 & 35.08 & 41.53 & 66.87 & 160.97 & 157.55 & 7.22 & 32.48 & 108.25 & 13.93 & 16.73 & 75.38 \\
\hline 2016 & $1122.6\{591.68$ & 494.34 & 36.67 & 44.12 & 60.55 & 166.87 & 173.70 & 6.65 & 33.90 & 127.14 & 18.31 & 11.17 & 88.99 \\
\hline 2017 & $1130.4 ؟ 597.30$ & 495.79 & 37.41 & 44.85 & 52.35 & 175.64 & 177.02 & 8.80 & 42.29 & 114.21 & 12.69 & 14.16 & 93.13 \\
\hline 2018 & $1062.5: 564.73$ & 457.88 & 39.94 & 37.45 & 55.31 & 164.94 & 174.46 & 7.34 & 32.13 & 110.05 & 14.44 & 16.90 & 86.58 \\
\hline
\end{tabular}




\section{Table S5: Yearly surface SO2 emissions in TgNyr-1.}

\begin{tabular}{|c|c|c|c|c|c|c|c|c|c|c|c|c|c|c|}
\hline $\operatorname{TgS} y^{-1}$ & Globe & $\mathrm{NH}$ & Tropics & $\mathrm{SH}$ & Europe & USA & C Africa & E China & S Africa & $\begin{array}{c}S \\
\text { America }\end{array}$ & N Africa & SE Asia & Australia & India \\
\hline 2005 & 36.33 & 25.91 & 7.24 & 3.18 & 2.87 & 2.86 & 0.69 & 7.00 & 0.50 & 0.42 & 0.66 & 0.13 & 1.01 & 1.51 \\
\hline 2006 & 35.54 & 25.17 & 7.11 & 3.26 & 2.94 & 2.52 & 0.59 & 7.54 & 0.49 & 0.37 & 0.58 & 0.13 & 1.13 & 1.63 \\
\hline 2007 & 39.04 & 27.65 & 7.99 & 3.40 & 2.87 & 2.73 & 0.75 & 8.25 & 0.48 & 0.46 & 1.13 & 0.13 & 1.17 & 1.54 \\
\hline 2008 & 37.12 & 27.00 & 6.89 & 3.24 & 2.90 & 2.88 & 0.60 & 7.70 & 0.39 & 0.30 & 0.45 & 0.13 & 1.13 & 1.84 \\
\hline 2009 & 35.50 & 24.87 & 7.09 & 3.54 & 2.95 & 2.64 & 0.66 & 6.09 & 0.50 & 0.28 & 0.47 & 0.15 & 1.35 & 1.66 \\
\hline 2010 & 34.65 & 23.76 & 7.48 & 3.40 & 3.03 & 2.38 & 0.71 & 5.85 & 0.56 & 0.47 & 0.53 & 0.13 & 1.21 & 1.74 \\
\hline 2011 & 39.13 & 27.74 & 7.89 & 3.51 & 3.21 & 2.48 & 0.69 & 6.89 & 0.43 & 0.29 & 0.69 & 0.13 & 1.54 & 2.19 \\
\hline 2012 & 35.43 & 24.32 & 7.72 & 3.40 & 2.79 & 2.33 & 0.74 & 6.45 & 0.52 & 0.34 & 0.77 & 0.12 & 1.28 & 1.74 \\
\hline 2013 & 34.28 & 23.62 & 7.20 & 3.46 & 2.89 & 2.42 & 0.70 & 5.74 & 0.50 & 0.29 & 0.61 & 0.11 & 1.27 & 1.79 \\
\hline 2014 & 33.76 & 22.70 & 7.65 & 3.41 & 2.67 & 2.54 & 0.74 & 4.61 & 0.50 & 0.34 & 0.61 & 0.12 & 1.28 & 1.86 \\
\hline 2015 & 33.25 & 21.88 & 7.76 & 3.61 & 2.70 & 2.42 & 0.74 & 4.11 & 0.57 & 0.40 & 0.60 & 0.13 & 1.31 & 1.88 \\
\hline 2016 & 32.09 & 21.26 & 7.30 & 3.53 & 2.67 & 2.39 & 0.60 & 3.79 & 0.58 & 0.40 & 0.57 & 0.14 & 1.24 & 2.01 \\
\hline 2017 & 33.05 & 21.80 & 7.66 & 3.59 & 2.70 & 2.51 & 0.56 & 3.62 & 0.60 & 0.52 & 0.48 & 0.15 & 1.31 & 2.25 \\
\hline 2018 & 31.58 & 20.65 & 7.49 & 3.44 & 2.68 & 2.46 & 0.58 & 3.46 & 0.58 & 0.49 & 0.56 & 0.12 & 1.18 & 1.93 \\
\hline
\end{tabular}




\begin{tabular}{|c|c|c|c|c|c|c|c|c|c|c|c|c|c|c|}
\hline $\mathrm{TgN}_{\mathrm{yr}} \mathrm{-1}^{-1}$ & Globe & $\mathrm{NH}$ & Tropics & $\mathrm{SH}$ & $\stackrel{\mathrm{N}}{\text { America }}$ & $\begin{array}{c}\mathrm{S} \\
\text { America }\end{array}$ & S Africa & N Africa & Siberia & India & SE Asia & Pacific & Atlantic & Australia \\
\hline 2005 & 6.80 & 2.32 & 3.69 & 0.79 & 0.48 & 0.98 & 0.80 & 0.74 & 0.51 & 0.06 & 0.77 & 0.19 & 0.02 & 0.21 \\
\hline 2006 & 7.08 & 2.37 & 3.87 & 0.84 & 0.50 & 1.07 & 0.90 & 0.66 & 0.55 & 0.05 & 0.86 & 0.17 & 0.01 & 0.26 \\
\hline 2007 & 7.61 & 2.55 & 4.19 & 0.87 & 0.52 & 1.11 & 0.83 & 0.73 & 0.58 & 0.06 & 0.98 & 0.21 & 0.03 & 0.26 \\
\hline 2008 & 7.72 & 2.44 & 4.39 & 0.89 & 0.43 & 1.25 & 0.90 & 0.73 & 0.53 & 0.06 & 1.06 & 0.22 & 0.02 & 0.28 \\
\hline 2009 & 7.88 & 2.54 & 4.38 & 0.96 & 0.50 & 1.34 & 0.82 & 0.75 & 0.58 & 0.06 & 1.04 & 0.23 & 0.02 & 0.25 \\
\hline 2010 & 7.45 & 2.49 & 4.07 & 0.89 & 0.40 & 1.04 & 0.75 & 0.72 & 0.57 & 0.06 & 0.98 & 0.24 & 0.03 & 0.32 \\
\hline 2011 & 7.70 & 2.35 & 4.36 & 0.99 & 0.39 & 1.28 & 0.81 & 0.70 & 0.58 & 0.07 & 0.98 & 0.26 & 0.02 & 0.36 \\
\hline 2012 & 7.38 & 2.53 & 3.89 & 0.96 & 0.50 & 1.07 & 0.74 & 0.66 & 0.56 & 0.07 & 0.88 & 0.28 & 0.02 & 0.27 \\
\hline 2013 & 7.33 & 2.43 & 3.97 & 0.93 & 0.46 & 1.14 & 0.69 & 0.67 & 0.57 & 0.07 & 0.87 & 0.28 & 0.03 & 0.26 \\
\hline 2014 & 7.63 & 2.58 & 4.09 & 0.97 & 0.54 & 0.99 & 0.81 & 0.74 & 0.57 & 0.07 & 0.93 & 0.27 & 0.03 & 0.28 \\
\hline 2015 & 7.13 & 2.44 & 3.75 & 0.94 & 0.55 & 0.94 & 0.77 & 0.69 & 0.53 & 0.07 & 0.88 & 0.28 & 0.03 & 0.22 \\
\hline 2016 & 7.67 & 2.62 & 4.05 & 1.01 & 0.44 & 1.05 & 0.75 & 0.76 & 0.58 & 0.07 & 1.06 & 0.27 & 0.02 & 0.24 \\
\hline 2017 & 7.76 & 2.47 & 4.33 & 0.97 & 0.45 & 1.20 & 0.76 & 0.82 & 0.54 & 0.05 & 1.08 & 0.23 & 0.03 & 0.25 \\
\hline 2018 & 7.86 & 2.59 & 4.31 & 0.96 & 0.55 & 1.20 & 0.79 & 0.71 & 0.58 & 0.06 & 1.08 & 0.26 & 0.02 & 0.26 \\
\hline
\end{tabular}

